\title{
Hybrid Approach for Annotating Unstructured Document
}

\author{
Meghana.H.J \\ PG Scholar, Dept., of CS \& E \\ Adichunchanagiri Institute of Technology, \\ Chikmagalur, Karnataka, India
}

\author{
Pushpa Ravikumar, B.E., M.Tech., Ph.D \\ Prof and HOD, Dept., of CS \& E \\ Adichunchanagiri Institute of Technology \\ Chikmagalur, Karnataka, India
}

\begin{abstract}
Annotation is a process of adding the information into the Document which is useful for extracting the information. A large number of organizations now days generate a large amount of data which is always present in the textual format. But such collections of textual document which contains a large amount of structured information which is completely hidden in the unstructured information. Information extraction algorithm is too costly because it always works on the top of the text and it does not provide the necessary structured information. In our paper, we present a method to generate the structured attribute by identifying the documents which contain the information of interest and this information in future useful for querying the database. The major contribution of this paper, we propose the algorithm, where it identifies the structured attribute which is present in the document by combining both the query workload and the content of the text document. Our Experiment result shows that our technique gives the better results compared to the methods which only relay on the content of the document and only on the query workload.
\end{abstract}

\section{General Terms}

Data mining, Annotating the text Document

\section{Keywords}

Annotation, CADS form, CV and QV

\section{INTRODUCTION}

Annotating the document is the comments, text and explanations which are added to the part of the document or the whole document. User can create and share the necessary information in many application purpose domains including social networking site, Disaster Management System. Information sharing tool like Microsoft share point allow user to create and share the document and also to tag them. In the same way user can define attributes for their objects in Google base. Hence annotation process can helpful for information discovery. Many annotation systems already contain "untyped" keyword annotation: for example, a user may tag the weather report using the annotation "StromName Gustav".

Attribute-value pairs for annotation are expensive because they contain more information compared to untyped approach. In this scenario, the above information can be entered as (StormName, Gustav). Many annotation systems that do not have the (attribute-value) annotation that makes use of "pay as you go"querying feasible. User should be principled in their effort for (attribute-value). Hence user should always know the underlying field type and schema they are using and also they should know when to use such fields.
We address CADS (Collaborative Adaptive Data Sharing platform), is an "annotate-the document" infrastructure which promote fielded data explanation to direct the annotation system our CADS system uses Query Workload, along with examining the content of the document. The goal of CADS is to lower the cost of annotated document which can be useful for the user given queries.

In this approach, the user generate a document what the user want to annotate and uploads it to the database repository. Then, CADS investigate the each content of the document and create an adaptive insertion form. This adaptive insertion form contains the information need (query workload), the best attribute names of the text document and possible attribute values given the document text.

\section{LITERATURE SURVEY}

In this section, related literature about various document annotation technique and scope of annotating the document will be reviewed and discussed.

M. J. Franklin, S. R. Jeffery and A. Y. Halevy [1], they proposed how to use the concept of value of perfect information (VPI) to order the candidate matches for user confirmation. They also proposed how efficiently they combine the utility function with the VPI to order user confirmation. An experiment result shows that how to evaluate both the real dataset and unreal dataset to order the user feedback which is produced by using the VPI based approach which yields a dataset with higher utility than a wide range of order utility. At last they summarize Roomba design, where a system uses the decision-theoretic framework to show the dataspace in request in a pay-as-you-go manner for user feedback .

But the assumption of their work is to match the query which contain the attribute with the attributes having the source because the data sources already contain structured data information and also to deal with imperfect user feedback. But they assumed that users answered correctly every time because matches may be different or challenging to answer correctly.

A. Jain and P. G. Ipeirotis [2] they described how to revel different document retrieval strategies which affect the quality of the extracted relation by using the analytical models. They also showed how to display Receiver Operating Characteristic (ROC) curves which is used to calculate the quality of extraction in robust way and to present how to use ROC analysis in a principled way which is used to select the extraction parameters. 
The assumption of this project is a large number of structured data is completely hidden in unstructured data and to get the targeted information from the document, they use automated information extraction algorithms and fact is that only documents that actually contain the related information and there exist a false positive when documents what the user want to process which do not contain the targeted information when they use information extraction algorithms which is automatic to extract such fields, which may affect the quality of the data.

M. Jayapandian and H. Jagadish [3] they defined how to generate the good set of forms by using the automated technique and to maximize the ability, whenever user ask the queries for forms-based interface has to bound both the number of forms generated and the difficulty of any one of the form.

J.M. Ponte and W.B. Croft [4] proposed a paper where they are consider this information retrieval scenario and proposed a solution to analyze the content. Based on probabilistic language modeling they proposed a approach for data retrieval. Their approach to modeling which integrates document indexing and non-parametric for document retrieval into a single model. But the assumption is not warranted using the similarity of document.

G. Das, M. Miah, H. Mannila and, V. Hristidis[5] define a paper which presents a paper using Integer Programming formulation in order to extract algorithm. But for processing for the small workload it takes significant amount of time but it gives optimal and nearest solution.

\section{METHODOLOGY}

System architecture is the theoretical design that defines the structure and behavior of a system. An architecture description is organized in a way that supports analysis about the structural properties of the system. System components or building blocks of the system are defined and provides a plan, by using this a products can be obtained, and systems are developed, that are work together to implement the overall system. The System architecture is shown below in the Fig 1 .

Here user uploads the documents in the document uploader which already contains the annotated document and the new document to annotate, where analyzer retrieves the documents and calculates the content value, query value for the attributes and also combines both the content and query value for the attributes, where it sort the attribute values in descending order and recommend the attribute with highest value and generate the new insertion form. This insertion form contains the best attribute for the new unstructured document, here the user may also add the new attributes for the default attribute and generate the new document upload form.

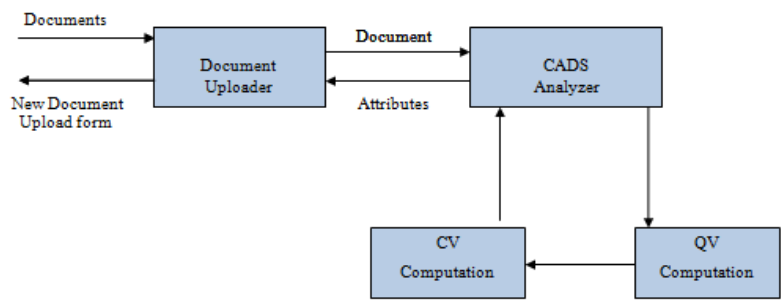

Fig 1: System architecture for document annotation.

\subsection{Use case Diagram for document Upload system}

Fig 2 shows the Use case diagram for document upload and to fill the annotation form. The System involves two use cases and a single user. Each of the two use cases represents a functionality provided to the system. The set of use cases shows the complete functionality of the system by the user module.

Fig 3 shows the Use case diagram to suggest the attribute to annotate the Document. The System involves five use cases and a single analyzer. Each of the five use cases represents a functionality provided to the system. The set of use cases shows the complete functionality of the system at high level of detail.

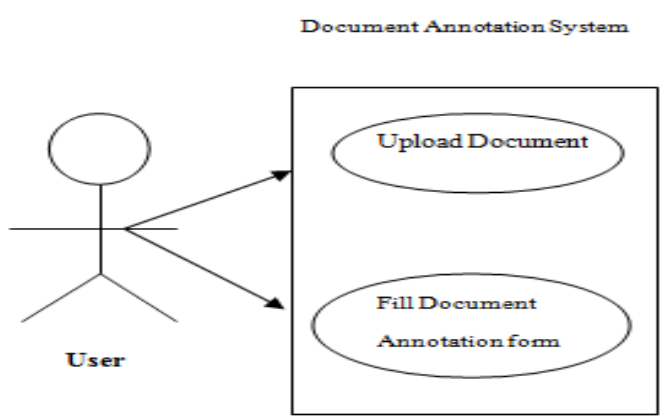

Fig 2: Use case diagram for document upload and fill Document Annotation Form.

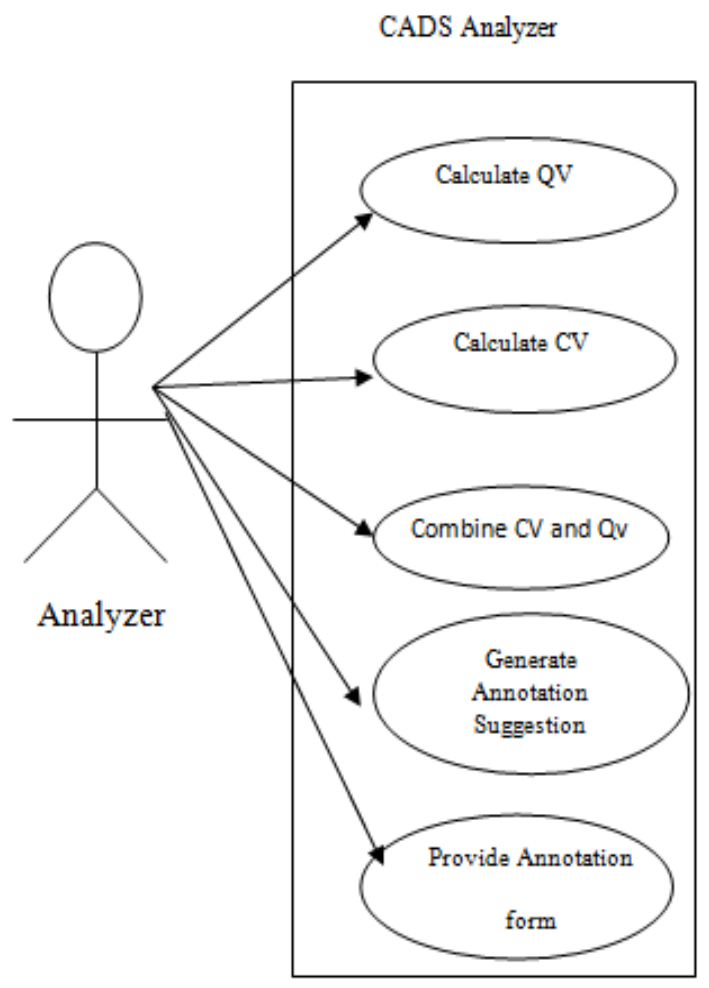

Fig 3: Use Case Diagram to suggest the attribute for the document 
The analyzer participates in the first and second use case by calculating the query value for the attribute based on the query posed by the user and also calculates the content value for each word in the text document what the user want to annotate with respect to attribute. In the third use case the analyzer combines both the content and query value with respect to attribute. Finally in fourth and fifth use case analyzer suggest the attribute based on the priority wise and generate the annotation form.

\section{EXPERIMENTAL RESULTS AND}

\section{ANALYSIS}

Implementation it is one of the phase of a project where the working system is turned out by using the theoretical design and it is a step where the system goes for actual functioning.

Here the contribution of our project work is the "attribute suggestion" problem, which applicable only for queries present in the query workload, and identifies the attributes that are present in the document, but not their values. We suggest the two properties for identifying and suggesting attributes for a document. They are:

1 With respect to the query workload $W l$, the attributes must have high querying value.

2 With respect to $d x_{0}$, the attributes must have high content value.

By adopting the probabilistic framework, we redefine the Attributes Suggestion problem as Probabilistic Attribute Suggestion which is defined above. Given $W$ (Workload) and $d$ (document) as the forecasts from different sources of evidence, our system (CADS) is the decision manager, with a specific prior $P$. we experiment it with two approaches.

We combine both objectives, in a principled way, using a probabilistic approach. Hence we experiment with two,

1. We combine the information from the forecasters assuming conditional independence, given $\mathrm{A}_{\mathrm{j}}$

$$
\operatorname{Score}\left(a_{t}\right)=\frac{p\left(a_{t} \mid w l\right)}{\left.1-p\left(a_{t} \mid w l\right)\right)} \cdot \frac{p\left(d x_{t} \mid a_{t}\right)}{p\left(d x_{t} \mid \overline{a_{t}}\right)}
$$

2. We build an approach that assumes conditional independence among the forecasters.

$$
p\left(a_{t} \mid w l, d x_{t}, p r\right)=\beta_{1} \cdot p\left(a_{t} \mid w l\right)+\beta_{2} \cdot p\left(a_{t} \mid d x_{t}\right)
$$

\subsection{QV, CV Computation and Combining Algorithm:}

The algorithm executes as follows:

1) Retrieve each attribute $A_{j}$ from $L_{Q V}$, where $L_{Q V}$ is the list of query values

2) Calculate the content Value (CV) for each attribute.

3) For each content value, where $\mathrm{CV}$ is the maximum value for the attribute which is unseen and Query value of the attribute is represented using $\operatorname{QV}\left(\mathrm{A}_{\mathrm{j}}\right)$, Calculate the Threshold value $\tau=F\left(C V, Q V\left(A_{j}\right)\right)$.

4) Add the attribute to $R$ if possible, where $R$ be highest score of the set of $\mathrm{k}$ attributes.

5) if the Threshold value $\tau<\operatorname{Score}\left(A_{k}\right)$, where $A_{k}$ is $k$-th attribute, Then return $\mathrm{R}$

Else go to Step 1

\subsection{Results and Discussions}

By clicking the method button which is already shown in the window, it consists of 3 module boxes one is conditional independence Equation 1, conditional independence Equation 2 and combine both $\mathrm{CV}$ and QV. On clicking the conditional equation 1 button the score of the entire attribute is calculated by using the Bayes equation which is given above and hence the window which is shown in the Fig 4 recommend the attribute results what the user uploaded the document to annotate.

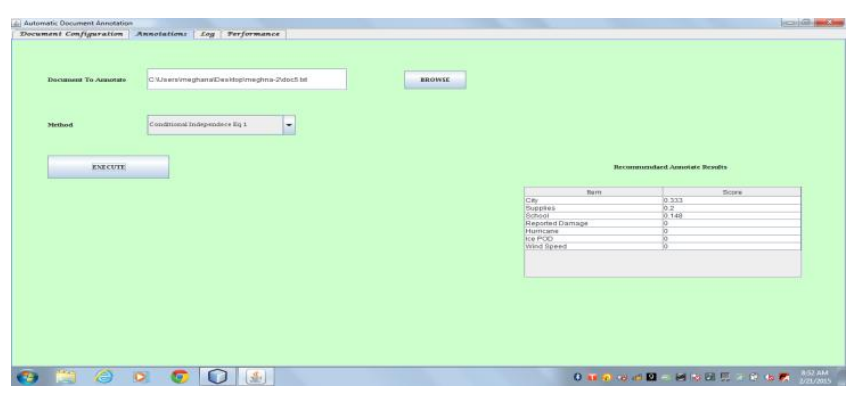

Fig 4: Results for calculating the CV and QV using conditional independence among attribute

By clicking the method button which is already shown in the below window, it also consists of 3 module boxes one is conditional independence equation 1, conditional independence equation 2 and combine both $\mathrm{CV}$ and QV. On clicking the conditional equation 2 button the score of the entire attribute is calculated by using the Bernoulli's equation which is given above and hence the window is shown in the Figure 5 recommend attribute results what the user uploaded the document to annotate.

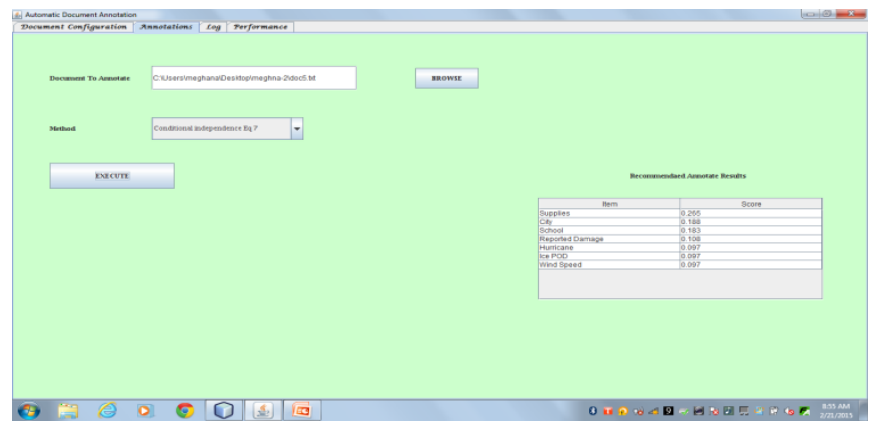

Fig 5: Results for calculating the $C V$ and $Q V$ using conditional independence among Forecaster

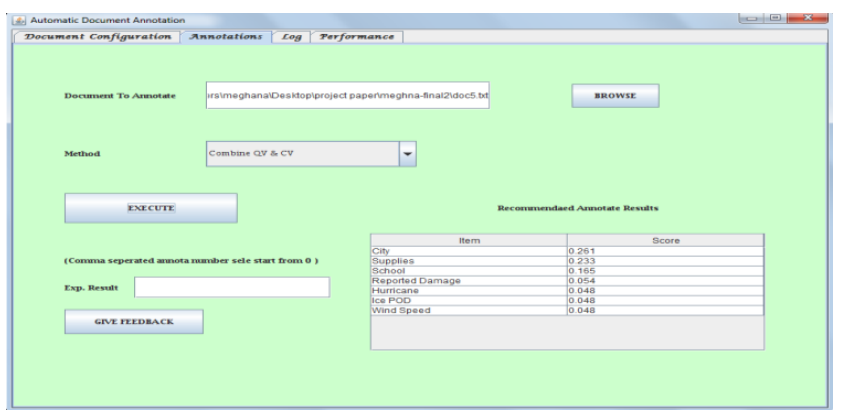

Fig 6: Combine $\mathrm{CV}$ and Qv value in sorted order 
The above Fig 6 represents the how efficiently we combine both the content and query value for the atribute. Hence this Fig 6 suggests the recommended attribute for the document.

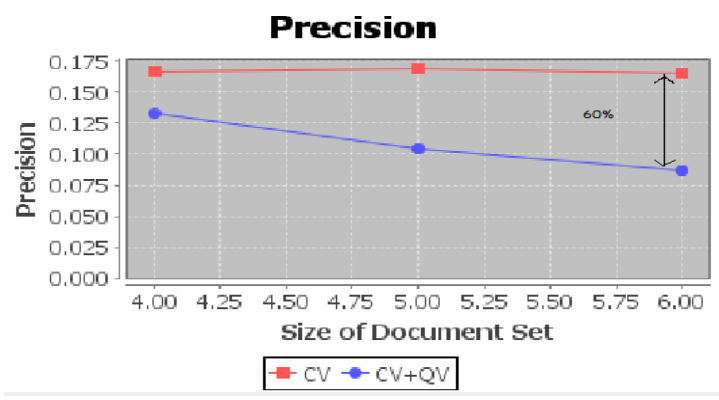

Fig 7: Graph generated for the variations in precision of suggested Attributes for the Document Annotation system

The above Fig 7 shows the precision graph which is obtained for the our project where variation of the suggested attribute precision is shown, here precision changes when the size of the document set increases. Here the proposed work increases their quality, when the documents set increases. The QV line is constant here, because it uses only the query workload.

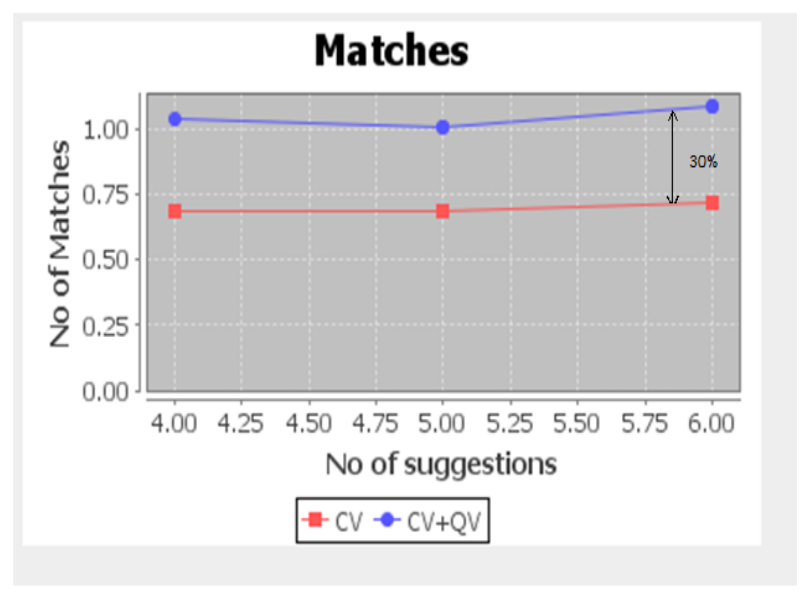

Fig 8: Graph generated for the Number of Query matches generated for the Document Annotation system
The above Fig 8 represents the graph which is generated for partial match for the attributes. To obtain this, where we count the queries which are satisfied by the documents, where we view each query condition as the queries.

\section{CONCLUSION}

In this paper a Collaborative Adaptive Data sharing Platform to suggest the relevant attributes to annotate the document to satisfy the user query. They provide a based by using a probabilistic methods which consider content of the document and query workload as the forecaster. So to combine these two pieces of evidence they are using the content and the query value. Experimental results shows that by using our techniques, the attribute can be suggested to improve the quality of searching by giving the user queries and also increase the visibility of the document.

\section{REFERENCES}

[1] S. R. Jeffery, M. J. Franklin, and A. Y. Halevy, "Pay-asyou-go user feedback for dataspace systems," in ACM SIGMOD, 2008.

[2] A. Jain and P. G. Ipeirotis, "A quality-aware optimizer for information extraction," ACM Transactions on Database Systems, 2009.

[3] M. Jayapandian and H. Jagadish, "Expressive query specification through form customization," in Proceedings of the $11^{\text {th }}$ international conference on Extending database technology: Advances in database technology, ser. EDBT '08. New York, NY, USA: ACM, 2008, pp. 416-427

[4] J. M. Ponte and W. B. Croft, "A language modeling approach to information retrieval," in Proceedings of the 21st annual international ACM SIGIR conference on Research and development in information retrieval, ser. SIGIR '98. New York, NY, USA: ACM, 1998,

[5] R. Fagin, A. Lotem, and M. Naor, "Optimal aggregation algorithms for middleware," J. Comput. Syst. Sci., vol. 66, pp. 614-656, June 2003.

[6] G. Tsoumakas and I. Vlahavas, "Random k-labelsets: An ensemble method for multilabel classification," in Proceedings of the $18^{\text {th }}$ European conference on Machine Learning, ser. ECML '07. Berlin, Heidelberg: Springer-Verlag, 2007, pp.406-417 